\title{
Predictors and patterns of participant adherence to a cortisol collection protocol
}

\author{
Daniel Hall, BA[Research Assistant], \\ School of Nursing, University of North Carolina at Chapel Hill \\ Diane Blyler, PhD[Project Manager], \\ School of Nursing, University of North Carolina at Chapel Hill \\ Deborah Allen, MSN, RN[Doctoral Student], \\ School of Nursing, University of North Carolina at Chapel Hill \\ Merle H Mishel, PhD, RN, FAAN ${ }^{*}$ [Kenan Professor], \\ School of Nursing, University of North Carolina at Chapel Hill \\ Jamie Crandell, PhD[Research Assistant Professor], \\ School of Nursing, University of North Carolina at Chapel Hill
}

Barbara B Germino, PhD, RN[Research Professor], and

School of Nursing, University of North Carolina at Chapel Hill

Laura S Porter, PhD[Associate Professor]

Duke University Medical Center, Duke University

\section{Summary}

Background-Cortisol, a stress-related hormone, has been measured in many psychoimmunological studies via collection of saliva; however, patterns of participant adherence to protocol procedures are rarely described in the literature.

Objectives-In this paper we examine adherence to a cortisol morning rise collection protocol and explore its associations with demographic predictors and fatigue.

\begin{abstract}
Method-Participants included 262 breast cancer survivors enrolled in a National Institute of Nursing Research funded longitudinal intervention study (5R01NR010190, M. Mishel, P.I.). Selfreported times of salivary cortisol collection were recorded for each of 12 saliva samples. Adherence was assessed with respect to various demographic factors and fatigue. Participants were categorized as having high, moderate, or low adherence to the saliva collection protocol.
\end{abstract}

Results-Overall, 117 (45\%) participants had high adherence to the protocol, 117 (45\%) participants had moderate adherence, and $28(\sim 11 \%)$ participants had low adherence. Tests for proportionality for the polytomous logistic regression indicated that demographic predictors in our model had a similar association with each level of participant adherence. Women who did not adhere to the saliva collection were more likely to be African American (OR .50, CI .29- .88) and

(C) 2010 Elsevier Ltd. All rights reserved.

*Corresponding Author: Merle Mishel, School of Nursing, CB\# 7460, University of North Carolina at Chapel Hill, Chapel Hill, NC, 27599 (:mishel@email.unc.edu).

Publisher's Disclaimer: This is a PDF file of an unedited manuscript that has been accepted for publication. As a service to our customers we are providing this early version of the manuscript. The manuscript will undergo copyediting, typesetting, and review of the resulting proof before it is published in its final citable form. Please note that during the production process errors may be discovered which could affect the content, and all legal disclaimers that apply to the journal pertain. 
to report a high impact of fatigue on their behaviors (OR .88, CI .79- .98). Though other predictors in the model were not statistically significant (working full time and living with at least one child under 18 years of age), the overall model was significant $\left(\chi^{2}(4)=17.35, p<.01\right)$.

Discussion-To our knowledge, this is the first study to examine profiles of participant adherence to a cortisol sampling protocol over multiple timepoints. By conceptualizing adherence as a polytomous outcome, future studies may give us insights into adherence trends in other populations with the aim of promoting adherence and designing more informed saliva collection protocols.

\section{Keywords}

cortisol; morning rise; adherence; saliva collection protocols

\section{Background}

Cortisol, a hormone involved in neuroendocrine functioning, is the product of the hypothalamus-pituitary-adrenal (HPA) axis with normal diurnal patterns observed in daily functioning. Secreted in response to stressful situations, cortisol influences the body's ability to adapt to demanding situations (Tsigos \& Chrousos, 2002). Cortisol is present in saliva, making it relatively easy to collect, and serves as a useful biomarker to measure when attempting to determine one's responses to stress.

In healthy individuals, cortisol levels rise sharply upon awakening. Current evidence suggests that the peak in morning cortisol occurs approximately 30 minutes after awakening (Fries et al., 2009). This cortisol morning rise (CMR; Prussner et al., 1997) is computed as the difference between two salivary cortisol samples with the first sample taken upon awakening and the second sample taken within the 15 to 45 minute window following awakening (Cohen et al., 2006).

Salivary cortisol collection protocols have varied greatly from study to study. Protocols vary in numbers of sampling days, numbers of sampling times, and actual sampling times. For example, some investigators collected samples on one day (Kudielka et al., 2003), while others collected samples over the course of two or more days (Horwitz \& Horwitz, 1993). There is considerable variability in the recommended number of samples used to calculate CMR, ranging from two samples to as many as five (Bhagwagar et al., 2005; Portella et al., 2005). Additionally, the reported time of day that samples are taken varies from study to study. Bhagwagar and colleagues (2005) reported a high variability of cortisol collection times in relation to waking; the authors posit that reliable timing of the second cortisol collection would have led to more conclusive findings.

Little published research describes how investigators have addressed analyses of cortisol samples that are not recorded at precisely the instructed time (Adam \& Kumari, 2009). Most frequently these data are excluded from analyses or averaged over several days of sample collection if available (Cohen et al., 2006). Especially in longitudinal studies involving cortisol collection, it is plausible that subjects may fail to provide a sample that was collected in a way that adhered perfectly to instructed collection times (Cohen et al., 2006). Therefore, with so much variability in sampling protocols among studies of cortisol, issues of participant adherence need to be addressed.

Adherence has been shown to be related to CMR patterns in several studies. Kudielka et al. (2003) compared 47 participants' subjective ratings of adherence to a saliva sampling protocol to data gathered by an electronic monitoring system over the course of one day. "Adherent" participants were defined as those who always complied with the protocol and 
"nonadherent" participants were those who demonstrated any failure to comply with the full protocol. No predictors were associated with adherence; however, the adherent subjects had a significantly higher CMR than nonadherent subjects $(\mathrm{p}=.007)$. The authors concluded that subject precision in the collection and recording of salivary sampling is paramount to the measurement of CMR. Likewise, Broderick and colleagues (2004) used within-subjects analyses to describe the impact of adherent and nonadherent days on cortisol slopes while describing cortisol differences for healthy controls or those with fibromyalgia. Of the 66 participants, 30 had days of adherence mixed with days of nonadherence to the sampling protocol for the analyses. Within these 30 subjects, the only cortisol measurements that demonstrated a classic CMR were those samples in which the subjects adhered to the sampling protocol procedures.

While both of these studies demonstrate the importance of subject adherence for accurate cortisol responses, it remains unclear what predictors are associated with and impact adherence. For instance, having competing responsibilities or a lack of energy may inhibit one's ability to adhere to an early morning saliva collection protocol. In particular, fatigued participants may experience difficulty following protocol procedures in the morning. Although fatigue has been explored as a potential influencer on participant adherence, findings have been inconclusive (Decker et al., 2009).

Additionally, participants in studies involving the longitudinal collection of salivary cortisol have yet to be categorized in terms of their overall adherence. In this paper, we will explore adherence in terms of three adherence levels (high, moderate, or low) to a protocol in a longitudinal study, and whether that variability in adherence is associated with demographic predictors and fatigue.

\section{Methods}

This study focused on adherence to a saliva collection protocol among 263 female breast cancer survivors who were participating in a longitudinal intervention study at the University of North Carolina at Chapel Hill School of Nursing. Participants who completed the entire study collected nine days of cortisol, and thus had nine opportunities to adhere to the CMR protocol. One participant was excluded from this analysis because she only participated in one cortisol collection day. The remaining participants were 162 Caucasian and 100 African American survivors who were English speaking, between one and four years post-treatment for breast cancer (diagnosed at stages 1 through 4 ), and had no prior history of receiving treatment for any other form of cancer. Screening to exclude anyone with cognitive impairment was conducted at the time of recruitment. Women were eligible to participate if they were less than 50 years of age at the time of recruitment. At baseline, participants ranged in age from 24 to 51 years $(\mathrm{M}=43.9, \mathrm{SD}=4.8)$, had between 10 and 25 years of formal education $(\mathrm{M}=15.1, \mathrm{SD}=2.3)$, and had between one and five children $(\mathrm{M}=$ $2.1, \mathrm{SD}=.87)$. Participants averaged 2.8 years since their last treatment $(\mathrm{SD}=1.1)$. All participants received a small financial incentive, small gifts and information about cancer survivorship.

Data collection took place over three timepoints [T1 (baseline), T2 (6 months post baseline), and T3 (7 months post baseline)]. At each timepoint, participants were asked to provide saliva samples four times a day (before they got out of bed in the morning, $30-40$ minutes after waking, before their evening meal, and at bedtime) for three consecutive days. For the purposes of studying the CMR, only the first two samples on each day are of interest.

Data collectors instructed participants on proper saliva sampling techniques, including adherence to specific sampling times, which were reiterated on an instruction card given to 
each participant (see Figure 1). At each timepoint, participants were given a box containing 12 numbered vials. Attached to the box was a small yellow card with 12 spaces, numbered to match the vials. The participant was instructed to write the time she collected each sample on the card. Participants were told to skip a vial if they missed the corresponding sampling time. At the T1 data collection visit, the data collectors demonstrated the procedure and had the participant practice the saliva collection process under their guidance and observation.

Saliva was collected using the passive drool method which has been shown to produce quality samples (Salimetrics, 2009; Shirtcliff et al., 2001). Participants were provided Medication Events Monitoring System (MEMS) bottles filled with drool collection straws and were told that times of straw retrieval would be recorded by the MEMS caps, thereby reinforcing protocol adherence (Broderick et al., 2004; Kudielka et al., 2003). Within our sample, MEMS data suggested that many subjects withdrew all straws at the beginning of data collection or on a daily basis, resulting in numerous missing data points. Other studies investigating adherence have also reported missing data resulting from MEMS use (Ailinger, Black, \& Lima-Garcia, 2008; Llabre et al., 2006; Samet, Sullivan, Traphagen, \& Ickovics, 2001). Thus self-report of times for salivary collections were used in these analyses.

Data from self-report cards of sampling times were entered into a SAS database using double data entry procedures. The difference between the second sample time and the first was then calculated for each of the three days across all timepoints to determine overall adherence of our subjects to the CMR protocol.

\section{Description of Intervention in Larger Study}

We have included a brief overview of the intervention and control groups since we conducted an analysis reported in this paper on the relationship between adherence to the intervention and adherence to the saliva collection. The Younger Breast Cancer Survivor Uncertainty Management Intervention (YS-UMI) was a randomized study conducted with Caucasian and African American breast cancer survivors who were 50 years of age or younger and one to five years after treatment completion at the time of recruitment. Participants were randomly assigned to either the intervention or attention control condition. The intervention consisted of 4 focused calls by a trained Nurse Interventionist on an uncertainty management intervention designed for younger breast cancer survivors. An attention control condition of 4 calls by psychology graduate students on non-intervention topics was implemented to balance potential effects from a schedule phone discussion across groups. Callers in both the intervention and control groups followed a script, and all calls were recorded and reviewed by one of the investigators to ensure that specific guidelines for time, content, and caller role were followed. Participants in the control group received the intervention materials at the end of the study.

\section{Measures}

Demographic Information was collected at baseline in a Demographic Data Form. For the purposes of this paper, variables of interest were age, time since treatment, years of formal education, race, co-habiting status, employment status, reported income, insurance coverage, having children, and smoking status.

Fatigue is defined as having dimensions of timing, emotional symptoms, emotional meaning, impact on and distress related to activities of daily living, and was measured using the revised Piper Fatigue Scale (PFS; Piper et al., 1998).The revised PFS consists of 22 numerically scaled items that vary from generic (none to a great deal) to specific (able to concentrate to unable to concentrate). Factor analysis resulted in four dimensions of 
subjective fatigue (behavioral/severity, affective meaning, sensory, and cognitive/mood) with all factor loadings above .5. The standardized Cronbach's alpha was .92 or above for all subscales.

\section{Data Analyses}

Exploratory analyses were conducted to determine trends in protocol adherence. If the second sample was taken within 15-45 minutes after the initial morning sample, the participant was deemed "adherent" for that day. "High" adherence was defined as having $80 \%$ or more of morning saliva samples deemed adherent. This cutoff was selected after visually inspecting the distribution of participants' adherence across our sample (see Figure 2). Other studies examining adherence have used this method to categorize participants as well (see Inouye et al., 2003). Additionally, support for this cutoff exists in literature on medication adherence (Ediger et al., 2007). "Low" adherence was defined as having 20\% or less of morning saliva samples deemed adherent. "Moderate" adherence was thus defined as having between $20 \%$ and $80 \%$ of morning saliva samples deemed adherent.

Chi-square tests were used to examine whether any demographic variables were associated with adherence. Those demographic variables hypothesized to be related to adherence were included in a proportional odds model using PROC LOGISTIC in SAS (version 9.2). A proportional odds model was chosen because the outcome (adherence) was a three-level ordinal variable (high, moderate, or low).

\section{Results}

Analyses were conducted to determine trends in adherence at the participant level. Results indicated that 117 (45\%) participants had high adherence to the protocol, 117 (45\%) participants had moderate adherence, and $28(\sim 11 \%)$ participants had low adherence. Table 1 displays demographic and treatment-related statistics for each adherence group. To check for baseline differences between participants based on adherence, chi-square analyses on demographic variables were conducted. There were no significant differences between adherence groups on these key variables.

A proportional odds model was fit with adherence as the polytomous outcome and race, fatigue - behavioral/severity, employment (full-time vs. other), and child caregiving responsibilities (participant lived with at least one child under the age of 18 vs. not) as predictors. These variables were included in the model because they were expressed in different ratios across groups (see Table 1) or because they might help to elucidate barriers to adherence early in the morning. In order to have an adequate sample size for each condition, these variables were categorically grouped into dichotomous outcomes with the exception of fatigue. Adherence was used in the model as an ordinal outcome variable. A test for violation of the proportional odds assumption was not significant $\left(\chi^{2}=8.54\right.$, $\mathrm{df}=4$, $\mathrm{p}=.07$ ), supporting the use of proportional odds for this data. Tests of the deviance and Pearson chi-square goodness-of-fit statistics was not significant, indicating adequacy of fit.

Results from the logistic regression appear in Table 2. Race and fatigue were significant predictors of adherence (both at $\mathrm{p}=.02$ ). The point estimates indicated that women were less likely to adhere to the saliva collection protocol if they were African American (OR .50, CI . $29-.88$ ), reported a high impact of fatigue on their behaviors (OR .88, CI .79- .98), worked full-time (OR .79, CI .45-1.36), or lived with at least one child under 18 years of age (OR .65, CI .37-1.12). The model as a whole explained some of the variability in adherence behavior $\left(\chi^{2}(4)=17.35, \mathrm{p}<.01\right)$. 
An additional question of interest was whether adherence to the cortisol collection procedures was associated with adherence to the intervention/control calls. Both the intervention and control participants were supposed to receive a series of 4 phone calls. $80 \%$ of participants $(\mathrm{N}=209$, with no significant difference between the intervention and control groups) were able to be contacted for all 4 calls. We examined the association between adherence to receiving the intervention/control calls and adherence to the cortisol protocol, and found it to be statistically significant $\left(\chi^{2}((2)=7.68, p=.02)\right.$. In fact, of the participants who adhered to all 4 phone calls, almost half $(\mathrm{N}=102,49 \%)$ had high adherence to the cortisol collection, whereas among those with lower intervention adherence, only $28 \%$ $(\mathrm{N}=53)$ of had high adherence to the cortisol collection procedures.

\section{Discussion}

To our knowledge, this is the first study to examine profiles of participant adherence over time to a salivary cortisol sampling protocol. The issue of adherence is of particular pertinence to researchers, considering the cost and time of collecting samples and, in the case of cortisol, the narrow time frame in which meaningful samples can be collected. Especially in studies with small samples or limited diversity within those samples, a few non-adherent participants could significantly impact data analysis or generalizability of findings. In most cases, samples from non-adherent participants are simply excluded from analyses on CMR (Bennet et al., 2004; Cohen et al., 2006; Harville et al., 2007).

\section{Protocol Strengths}

Our saliva collection protocol had several strengths that aimed to maximize participant sampling collection adherence, including detailed explanations and a practice session with each participant, a simple saliva collection kit with clear instructions, use of the MEMS cap system containing the straws used for saliva collection, and data collector retrieval of saliva collection kit upon completion. Because data collection took place in each participant's home, the data collector was able to explain the saliva collection protocol, demonstrate the passive drool technique, and help the participant strategize how to best incorporate that protocol into her often busy life. Data collectors reminded participants to keep the saliva collection kits close to their beds at night, to refer to the instructions card, and to be sure to write down the time the participant did each sample.

The saliva collection kit was designed to be simple and straightforward to enhance participant adherence. In addition to the barcode that was affixed for the lab processing and tracking, each vial was clearly numbered with large numbers using a thick black marker and placed in order in the box. The numbers assisted the participant to know how many samples she had completed that day and also provided research staff with a way to know if a particular timepoint was skipped during the day. Lastly, data collectors retrieved the saliva collection kits from each participant, minimizing participant burden. In summary, our protocol had many strengths that we believe bolstered adherence in our sample; future studies replicating some of our methodology would be important.

\section{Predictors of Adherence}

In our model, two variables were significant predictors of adherence: race and fatigue. It is likely that "race" was capturing a complex array of conditions and issues impacting adherence to the saliva collection protocol. Race encompasses many factors of social inequality that have persisted over time in the United States (Williams et al., 2010). More research is needed to begin to identify and understand how these intersecting factors may create conditions that impact adherence. Few studies on the CMR have had diverse samples, and no studies that we are aware of have reported adherence to the collection protocol in 
terms of race. For instance, while Cohen and colleagues (2006) reported a large sample $(\mathrm{N}=781,58 \%$ female) with considerable minority participation (54\% African American), they did not report on differences in adherence between subgroups of participants. Inclusion criteria for acceptable times of cortisol sampling resulted in some samples being excluded from analysis; however, no information was reported about these participants. Indeed, there is a general lack of information on patterns of CMR in African Americans. In a recent review of CMR literature, Chida and Steptoe (2009) do not discuss race or ethnicity.

It is not surprising that fatigue was a significant predictor in the model. Prior research has found that both the intensity and duration of fatigue are significantly greater for women undergoing treatment for breast cancer when compared with healthy controls and may last as long as 10 years following a cancer diagnosis (Bower, 2008). It seemed plausible that participants in our study who were experiencing significant fatigue symptoms might also have difficulty adhering to a demanding saliva collection protocol, especially one that required the collection of the day's first sample upon awakening as well as a second sample 30-40 minutes later. A future intervention study might examine whether ameliorating fatigue can have a positive impact on participant adherence.

While other predictors in the model were not statistically significant, our findings indicate that it may be important to help parents who work full-time or who have caregiving responsibilities develop strategies to find ways to both complete the CMR collection timepoints and also meet their other competing responsibilities. Other family members, especially spouses/partners, may be able to assist with these competing responsibilities, especially if they are included in the discussion regarding the importance of both an immediate collection of saliva after waking and an accurate record of all times in the saliva collection protocol.

Finally, of note was the significant association between adherence to the intervention/control phone calls and adherence to cortisol collection. Participants who were high in phone call adherence were more likely to have high adherence to our cortisol collection protocol. This finding suggests that participants who adhere highly to one procedure in an intervention study may be more likely to adhere to cortisol collection procedures in that same study.

\section{Limitations}

This paper described adherence to a cortisol collection protocol among a sample of younger breast cancer survivors. Treatment-related sequelae and other factors associated with breast cancer survivorship limit the generalizability of our findings to other populations, such as healthy individuals or patients with other illnesses. Therefore, the results of this paper must be interpreted within the context of breast cancer survivorship.

One specific limitation noted in this study is that subjects were not asked to self-report their awakening time as compared to the time they collected their first morning sample. In this study, participants were asked to collect the first morning sample "before you get out of bed in the morning." It may have been better to have used language suggested by Cohen et al (2006) when they instructed participants to do the first saliva sample "when your eyes open and you are ready to get up" to collect the first saliva sample of the day. Future work should follow the recommendation of Adam and Kumari (2009) to define "waking" as clearly as possible.

In this study we used subject self-reported dates and times for this analysis. Strict monitoring of each sample collection in a lab was not possible because of the three-day, three times a day collection periods and the nature of this community-based intervention study. We recognize that collecting saliva samples in the lab would provide the best data 
regarding adherence to the collection protocol, but using subjects who are busy younger survivors with caregiving, work, and family responsibilities that make it imperative to collect data in a way and a place that is acceptable to them provides a true reflection of adherence issues.

Additionally, it is possible that medication may impact fatigue or adherence, and we did not control for the use of medications in these analyses. However, as Granger et al (2009) point out, there are some populations where medication use is the norm. Given that many of our participants are on Tamoxifen or other similar medications as part of the standard protocol for their breast cancer treatment, it would not be ethical to have them withhold their medications, and excluding them would greatly restrict the generalizability of our findings.

Finally, future research on adherence in cortisol collection may benefit from including a measure of study satisfaction. It may be the case that participants who feel satisfied at the completion of one cortisol sampling are more likely to adhere to collection protocols in subsequent samplings.

\section{Implications}

This study adds to the growing literature on saliva collection protocols and participant adherence by examining demographic predictors of adherence patterns and offering an approach to consider adherence as more than a dichotomous outcome. Of note in this large sample of breast cancer survivors, race and fatigue were significant demographic differences across adherence groups. Future research would benefit from investigating adherence to cortisol collection protocols with respect to other populations (Urizar et al., 2004) as well as examining protocol adherence as a polytomous outcome in non-cancer populations.

\section{References}

Adam EK, Kumari M. Assessing salivary cortisol in large-scale, epidemiological research. Psychoneuroendocrinology. 2009 In Press, Corrected Proof. doi: 10.1016/j.psyneuen.2009.06.011.

Ailinger RL, Black PL, Lima-Garcia N. Use of Electronic Monitoring in Clinical Nursing Research. Clinical Nursing Research 2008;17(2):89-97. doi: 10.1177/1054773808316941. [PubMed: 18387881]

Bennett GG, Merritt MM, Wolin KY. Ethnicity, education, and the cortisol response to awakening: a preliminary investigation. Ethnicity \& Health 2004;9(4):337-347. doi: 10.1080/1355785042000285366. [PubMed: 15570679]

Bhagwagar Z, Hafizi S, Cowen P. Increased salivary cortisol after waking in depression. Psychopharmacology 2005;182(1):54-57. doi: 10.1007/s00213-005-0062-z. [PubMed: 15991000]

Bower JE. Behavioral Symptoms in Patients With Breast Cancer and Survivors. Journal of Clinical Oncology 2008;26(5):768-777. doi: 10.1200/jco.2007.14.3248. [PubMed: 18258985]

Broderick JE, Arnold D, Kudielka BM, Kirschbaum C. Salivary cortisol sampling compliance: comparison of patients and healthy volunteers. Psychoneuroendocrinology 2004;29(5):636-650. doi: 10.1016/S0306-4530(03)00093-3. [PubMed: 15041086]

Chida Y, Steptoe A. Cortisol awakening response and psychosocial factors: A systematic review and meta-analysis. Biological Psychology 2009;80(3):265-278. doi: 10.1016/j.biopsycho.2008.10.004. [PubMed: 19022335]

Cohen S, Schwartz JE, Epel E, Kirschbaum C, Sidney S, Seeman T. Socioeconomic Status, Race, and Diurnal Cortisol Decline in the Coronary Artery Risk Development in Young Adults (CARDIA) Study. Psychosom Med 2006;68(1):41-50. doi: 10.1097/01.psy.0000195967.51768.ea. [PubMed: 16449410]

Decker V, Spoelstra S, Miezo E, Bremer R, You M, Given C, et al. A Pilot Study of an Automated Voice Response System and Nursing Intervention to Monitor Adherence to Oral Chemotherapy Agents. Cancer Nursing 2009;32(6):E20-E29. doi: 10.1097/NCC.0b013e3181b31114. [PubMed: 19816160] 
Ediger JP, Walker JR, Graff L, Lix L, Clara I, Rawsthorne P, et al. Predictors of Medication Adherence in Inflammatory Bowel Disease. American Journal of Gastroenterology 2007;102(7): 1417-1426. doi: 10.1111/j.1572-0241.2007.01212.x. [PubMed: 17437505]

Fries E, Dettenborn L, Kirschbaum C. The cortisol awakening response (CAR): Facts and future directions. International Journal of Psychophysiology 2009;72(1):67-73. doi: 10.1016/j.ijpsycho. 2008.03.014. [PubMed: 18854200]

Granger DA, Hibel LC, Fortunato CK, Kapelewski CH. Medication effects on salivary cortisol: Tactics and strategy to minimize impact in behavioral and developmental science. Psychoneuroendocrinology 2009;34(10):1437-1448. doi: 10.1016/j.psyneuen.2009.06.017. [PubMed: 19632788]

Harville EW, Savitz DA, Dole N, Herring AH, Thorp JM, Light KC. Patterns of salivary cortisol secretion in pregnancy and implications for assessment protocols. Biological Psychology 2007;74(1):85-91. doi: 10.1016/j.biopsycho.2006.07.005. [PubMed: 16979811]

Horwitz RI, Horwitz SM. Adherence to Treatment and Health Outcomes. Archives of Internal Medicine 1993;153(16):1863-1868. doi: 10.1001/archinte.1993.00410160017001. [PubMed: 8250647]

Inouye SK, Bogardus ST Jr, Williams CS, Leo-Summers L, Agostini JV. The Role of Adherence on the Effectiveness of Nonpharmacologic Interventions: Evidence From the Delirium Prevention Trial. Archives of Internal Medicine 2003;163(8):958-964. doi: 10.1001/archinte.163.8.958. [PubMed: 12719206]

Kudielka BM, Broderick JE, Kirschbaum C. Compliance With Saliva Sampling Protocols: Electronic Monitoring Reveals Invalid Cortisol Daytime Profiles in Noncompliant Subjects. Psychosom Med 2003;65(2):313-319. doi: 10.1097/01.psy.0000058374.50240.bf. [PubMed: 12652000]

Llabre MM, Weaver KE, Duran RE, Antoni MH, McPherson-Baker S, Schneiderman N. A measurement model of medication adherence to highly active antiretroviral therapy and its relation to viral load in HIV-positive adults. AIDS Patient Care \& STDs 2006;20(10):701-711. doi: 10.1089/apc.2006.20.701. [PubMed: 17052140]

Piper BF, Dibble SL, Dodd MJ, Weiss MC, Slaughter RE, Paul SM. The revised Piper fatigue scale: Psychometric evaluation in women with breast cancer. Oncology Nursing Forum 1998;25 677-68.

Portella MJ, Harmer CJ, Flint J, Cowen P, Goodwin GM. Enhanced Early Morning Salivary Cortisol in Neuroticism. Am J Psychiatry 2005;162(4):807-809. doi: 10.1176/appi.ajp.162.4.807. [PubMed: 15800161]

Pruessner JC, Wolf OT, Hellhammer DH, Buske-Kirschbaum A, von Auer K, Jobst S, et al. Free Cortisol Levels after Awakening: A Reliable Biological Marker for the Assessment of Adrenocortical Activity. Life Sciences 1997;61(26):2539-2549. doi: 10.1016/ S0024-3205(97)01008-4. [PubMed: 9416776]

Salimetrics. Saliva Collection and Handling Advice. State College, PA: Salimetrics, LLC; 2009. p. 1-14.Retrieved from http://www.salimetrics.com/assets/documents/all-things-saliva/Saliva-Collection-and-HandlingAdvice-large-format-4-7-09.pdf

Samet JH, Sullivan LM, Traphagen ET, Ickovics JR. Measuring Adherence Among HIV-Infected Persons: Is MEMS Consummate Technology? AIDS and Behavior 2001;5(1):21-30. doi: 10.1023/ A: 1009503320498.

Shirtcliff EA, Granger DA, Schwartz E, Curran MJ. Use of salivary biomarkers in biobehavioral research: cotton-based sample collection methods can interfere with salivary immunoassay results. Psychoneuroendocrinology 2001;26(2):165-173. doi: 10.1016/S0306-4530(00)00042-1. [PubMed: 11087962]

Tsigos C, Chrousos GP. Hypothalamic-pituitary-adrenal axis, neuroendocrine factors and stress. Journal of Psychosomatic Research 2002;53(4):865-871. doi: 10.1016/S0022-3999(02)00429-4. [PubMed: 12377295]

Urizar GG, Milazzo M, Le H-N, Delucchi K, Sotelo R, Muñoz RF. Impact of stress reduction instructions on stress and cortisol levels during pregnancy. Biological Psychology 2004;67(3): 275-282. doi: 10.1016/j.biopsycho.2003.11.001. [PubMed: 15294386] 


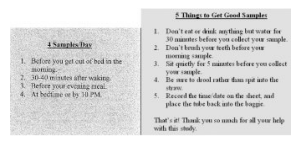

Figure 1.

Suggestions for Obtaining Adherent Saliva Samples (Front and Back of Card) 


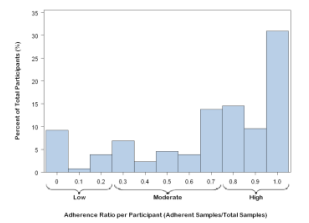

Figure 2.

Distribution of Adherence across All Participants ( $N=262)$ 
Table 1

Demographic and Health-Related Characteristics of Participants by Adherence $(\mathrm{N}=262)$

\begin{tabular}{|c|c|c|c|}
\hline & High & Moderate & Low \\
\hline$N$ (participants) & 117 & 117 & 28 \\
\hline Age (years) & $\mathrm{M}=44, \mathrm{SD}=4.7$ & $\mathrm{M}=44, \mathrm{SD}=4.8$ & $\mathrm{M}=44, \mathrm{SD}=5.1$ \\
\hline Years Since Tx & $\mathrm{M}=2.9, \mathrm{SD}=1.1$ & $\mathrm{M}=2.8, \mathrm{SD}=1.1$ & $\mathrm{M}=2.5, \mathrm{SD}=1.0$ \\
\hline Education (years) & $\mathrm{M}=15.0, \mathrm{SD}=2.1$ & $\mathrm{M}=15.4, \mathrm{SD}=2.5$ & $\mathrm{M}=14.4, \mathrm{SD}=2.5$ \\
\hline \multicolumn{4}{|l|}{ Race } \\
\hline White & $78(66.7 \%)$ & $75(64.1 \%)$ & $9(32.1 \%)$ \\
\hline African American & $39(33.3 \%)$ & $42(35.9 \%)$ & $19(67.9 \%)$ \\
\hline \multicolumn{4}{|l|}{ Marital Status } \\
\hline Married & $78(66.7 \%)$ & $81(69.2 \%)$ & $20(71.4 \%)$ \\
\hline Living with partner & $8(6.8 \%)$ & $11(9.4 \%)$ & $0(0 \%)$ \\
\hline Separated & $4(3.4 \%)$ & $5(4.3 \%)$ & $2(7.1 \%)$ \\
\hline Divorced & $11(9.4 \%)$ & $10(8.6 \%)$ & $5(17.9 \%)$ \\
\hline Widowed & $3(2.6 \%)$ & $0(0 \%)$ & $0(0 \%)$ \\
\hline Never Married & $13(11.1 \%)$ & $10(8.6 \%)$ & $1(3.6 \%)$ \\
\hline \multicolumn{4}{|l|}{ Co-Habiting Status } \\
\hline Live alone & $9(7.7 \%)$ & $9(7.7 \%)$ & $2(7.1 \%)$ \\
\hline Husband/Partner & $29(24.8 \%)$ & $33(28.2 \%)$ & $10(35.7 \%)$ \\
\hline Husb/Part + Children & $57(48.7 \%)$ & $56(47.9 \%)$ & $10(35.7 \%)$ \\
\hline Children only & $2(1.7 \%)$ & $8(6.8 \%)$ & $2(7.1 \%)$ \\
\hline Other & $20(17.1 \%)$ & $11(9.4 \%)$ & $4(14.3 \%)$ \\
\hline \multicolumn{4}{|l|}{ Employment Status } \\
\hline F/T Employed & $73(62.4 \%)$ & $68(58.1 \%)$ & $15(53.6 \%)$ \\
\hline P/T Employed & $16(13.7 \%)$ & $22(18.8 \%)$ & $3(10.7 \%)$ \\
\hline Other ${ }^{*}$ & $28(23.9 \%)$ & $27(23.1 \%)$ & $10(35.7 \%)$ \\
\hline \multicolumn{4}{|l|}{ Reported Income (monthly) } \\
\hline Less than $\$ 500$ & $1(0.9 \%)$ & $3(2.6 \%)$ & $1(3.7 \%)$ \\
\hline$\$ 500-\$ 1000$ & $6(5.2 \%)$ & $5(4.4 \%)$ & $2(7.4 \%)$ \\
\hline$\$ 1001-\$ 2000$ & $18(5.5 \%)$ & $19(16.5 \%)$ & $7(25.9 \%)$ \\
\hline$\$ 2001-\$ 4000$ & $36(31 \%)$ & $32(27.8 \%)$ & $7(25.9 \%)$ \\
\hline More than $\$ 4000$ & $55(47.4 \%)$ & $56(48.7 \%)$ & $10(37 \%)$ \\
\hline \multicolumn{4}{|l|}{ Insurance Coverage } \\
\hline Medicare/ Medicaid/ Disability & $5(4.3 \%)$ & $11(9.4 \%)$ & $1(3.6 \%)$ \\
\hline Private/HMO/PPO & $106(90.6 \%)$ & $101(86.3 \%)$ & $23(82.1 \%)$ \\
\hline None & $6(5.1 \%)$ & $5(4.3 \%)$ & $4(14.3 \%)$ \\
\hline
\end{tabular}




\begin{tabular}{lrrr}
\hline & High & Moderate & Low \\
\hline Number of Children & & & \\
$1-2$ & $94(80.3 \%)$ & $97(82.9 \%)$ & $22(78.6 \%)$ \\
3 or more & $23(19.7 \%)$ & $20(17.1 \%)$ & $6(21.4 \%)$ \\
\hline Smoking Status & & & \\
Smoker & $12(10.3 \%)$ & $9(7.7 \%)$ & $3(10.7 \%)$ \\
Non-smoker & $105(89.7 \%)$ & $108(92.3 \%)$ & $25(89.3 \%)$ \\
\hline
\end{tabular}

Note: A few participants did not provide information on all demographic variables.

*"Other" included participants who were on medical leave, retired, domestic caretakers, unemployed, or who responded "Other". 
